\title{
DETERMINANTS OF WATER USERS ASSOCIATION FORMATION AND THEIR RELATIONSHIP WITH CARRYING OUT ITS TASKS, AFIELD STUDY ON EL- MANAIFA CANAL FARMERS AT KAFER EL-SHIEKH GOVERNORATE
}

Hegazy, H. M. E.

Agric. Extension \& Rural Development Research Inst . ARC

\section{محددات تشكيل روابط مستخدمي الميـاه وعلاقتهـا بالقيام بمهامهـا دراسـة ميدانيـة على مزارعي ترعـة المنايفة بمحافظة كفر الشيخ

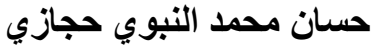 معهز بحوث الإرشاد الزراعي و والتنمية الريفيةــ مركز البحوث الزراعية الملخص}

استهدف هذا البحث بصفة أساسية التعرف على محددات تشكيل روابط مستخدمي المياه و علاقتها بالقيام بمهامها ولتحقيق ذلك تم وضئ وضع ثلاثة

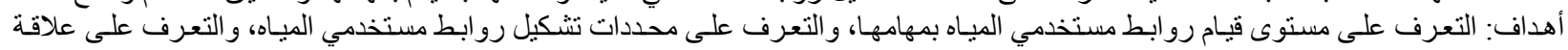

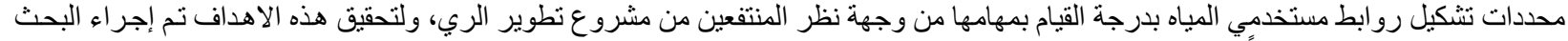

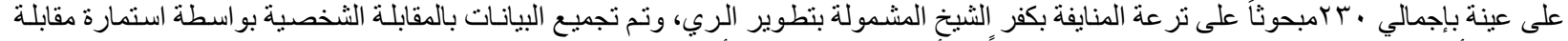

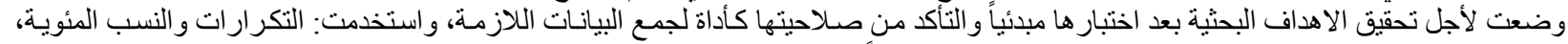

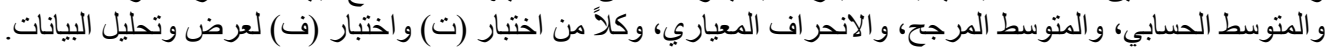
وقد توصل البحث إلى النتائج الآتية :

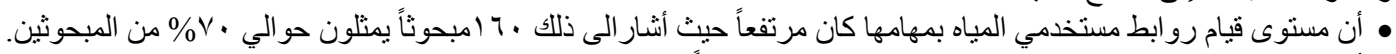

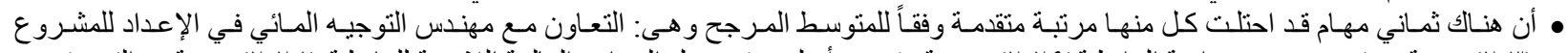

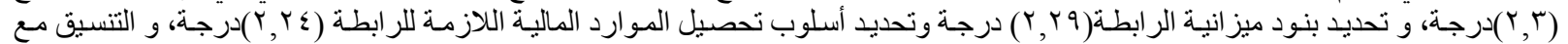

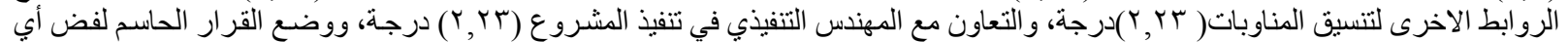

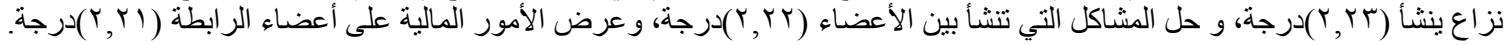

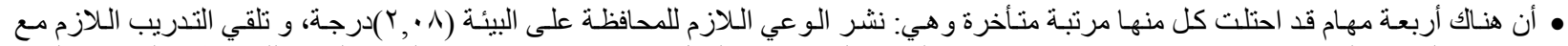

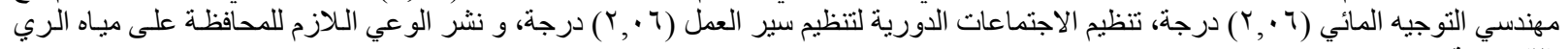
(r) (r) درجة. • وجود فروق معنة دروية في درجة قيام رو ابط مستخدمي المياه من القيام بمهامها بين المبحوثين عند تصنيفهم وفقا لمحددات تشكيل رو ابط مستخدمي المياه المدروسة.

المروية والتي تمثل ^. 99\% من اجمالي المساحة المنزر عة لإنتاج الغذاء

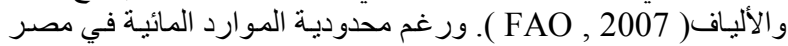

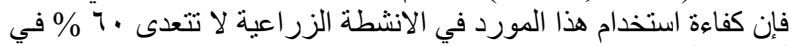

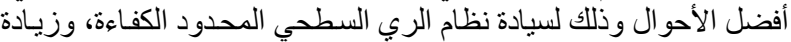

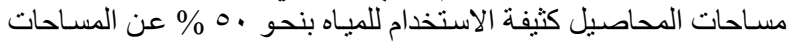

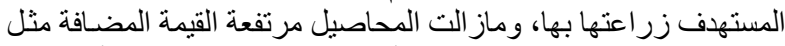

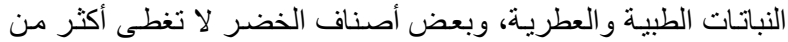

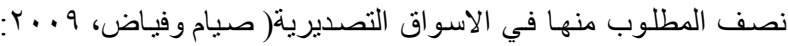

( ( $\left.1 \Lambda_{-}\right) \mathrm{V}$

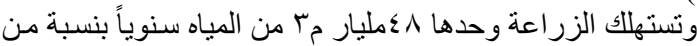

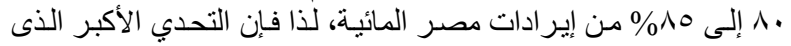

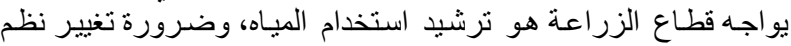

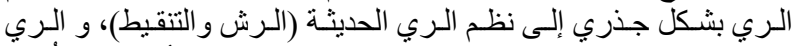

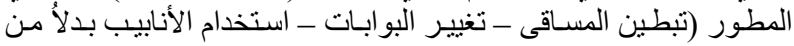

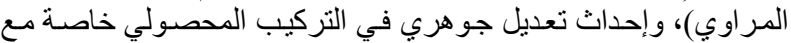

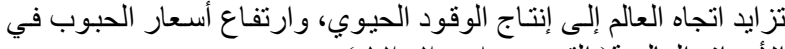

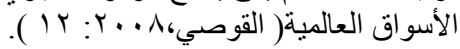

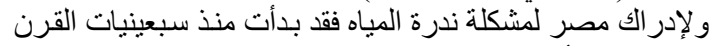

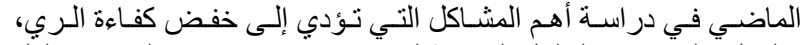

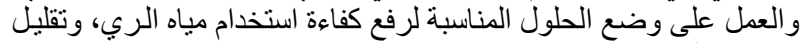

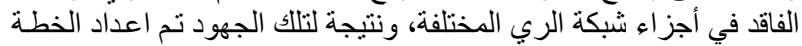

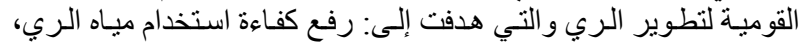

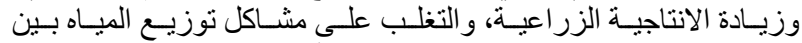

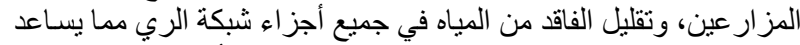

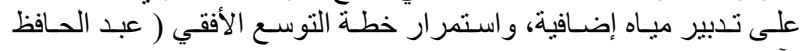

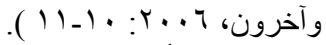

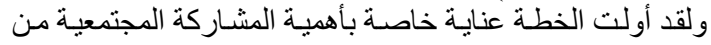

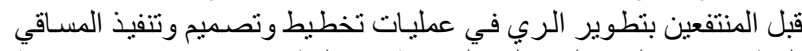

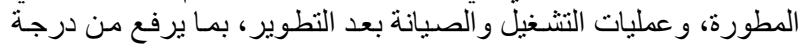

\section{المقدمة والمشكثة}

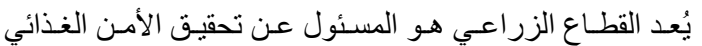

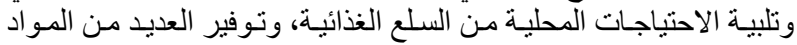

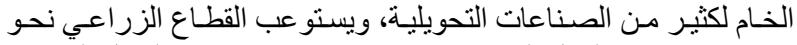

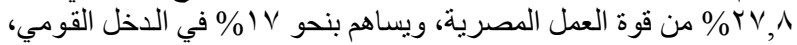

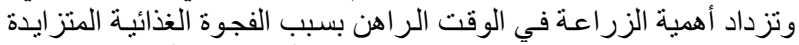

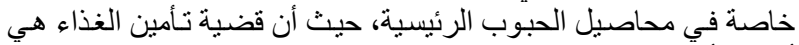

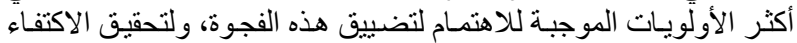

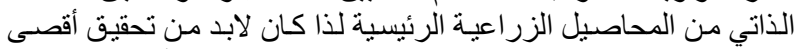

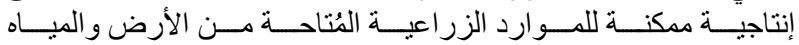

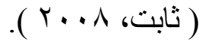

وتعتبر محدودية الموارد المائية المتاحة لمصدر عقبة حقدية حقيقية أمسام

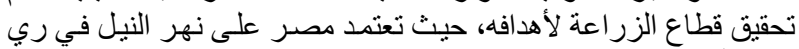

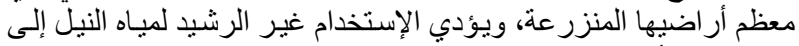

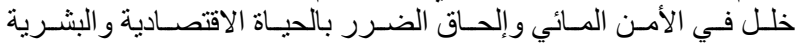

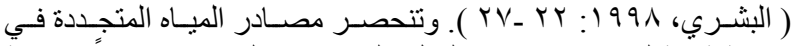

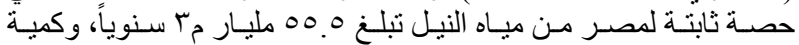

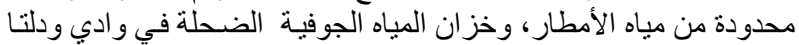

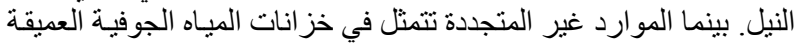

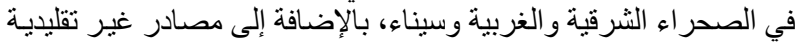

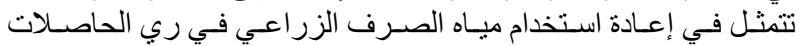

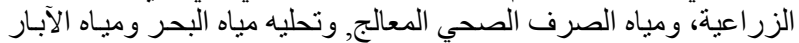

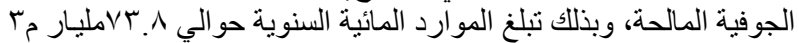
من مختلف مصادر ها (Gad\&Ali,2009: 302).

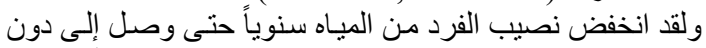

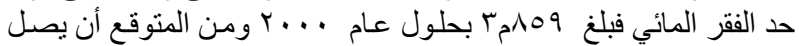

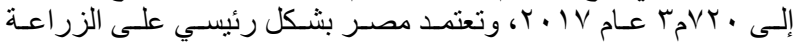


الفرض البحثي :

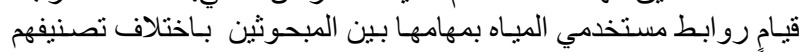

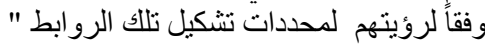

\section{الطريقة البحثية}

ا ـ شاملة وعينة البحث: أجري هذا البحث على تر عـة المنايفة بمحافظة

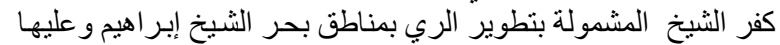

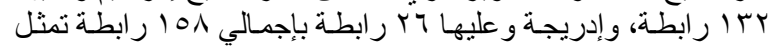

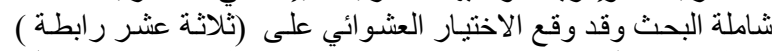

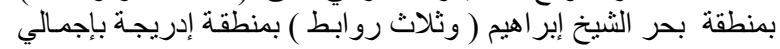

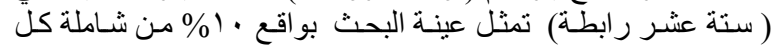

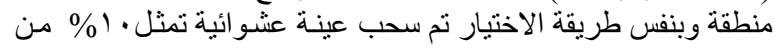

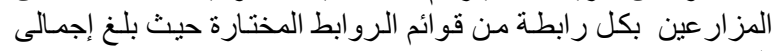

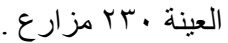

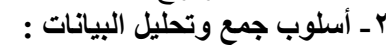

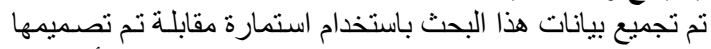

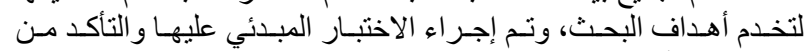

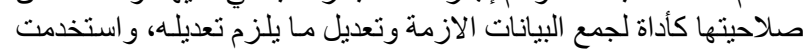

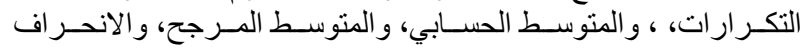

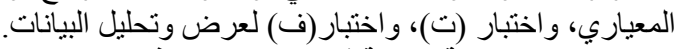

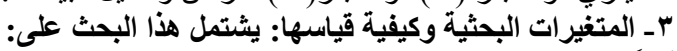

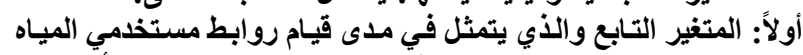

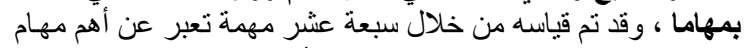

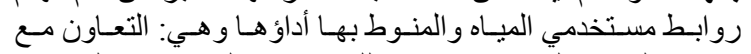

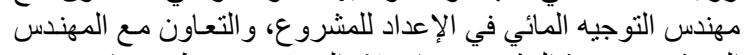

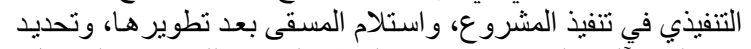

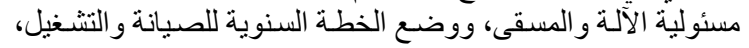

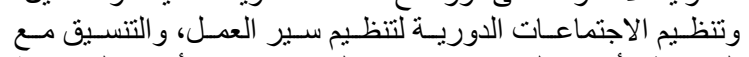

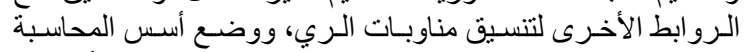

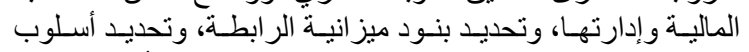

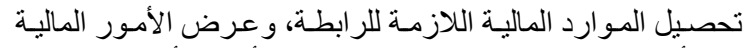

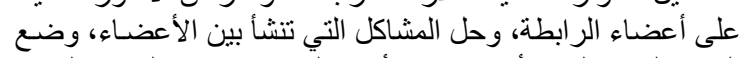

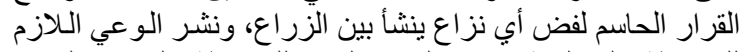

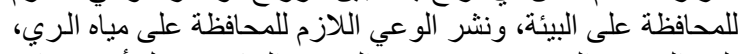

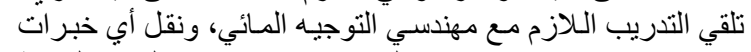

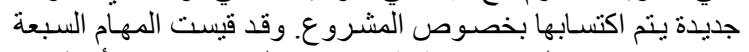

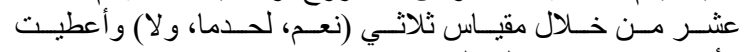

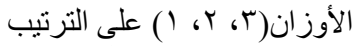

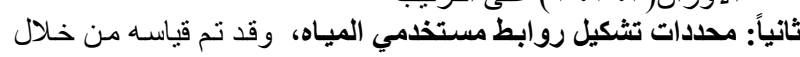

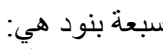

ا -مشاركة المبحوثين في الإجر اءات التحضيرية لعمل الر ابطة.

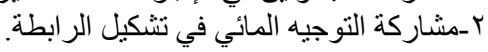

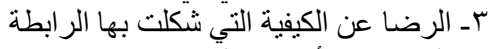

عـ التو افق بين أعضاء الرفيفة الريطة.

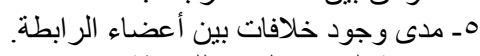

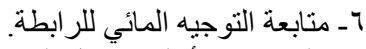

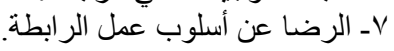

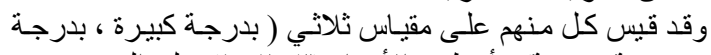

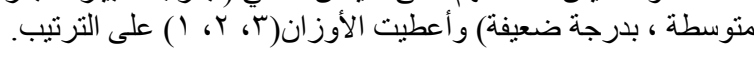

\section{النتائج البحثية ومناقشتها وتفسيرها}

وتثثمل عرض لأهم النتائج البحثية التي تم التوصل إليها وفقاً لأهداف وملفئ

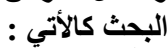
أولاً: النتائج التي تتعلق بمستوى قيام روابط مستخدمي المياه بمهامها.

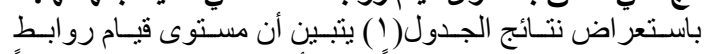

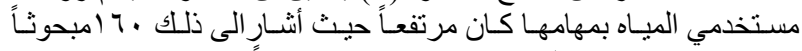

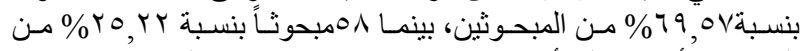

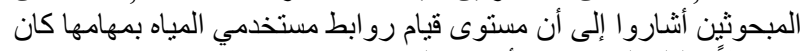

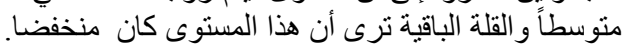

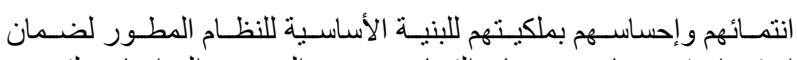

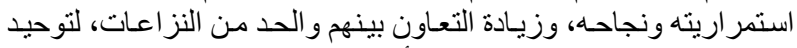

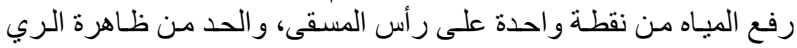

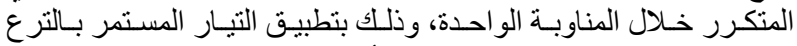

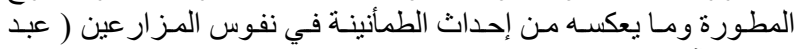

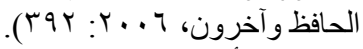

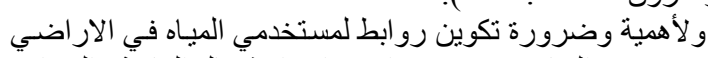

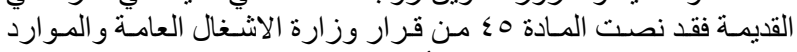

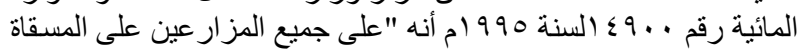

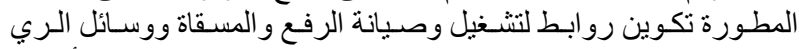

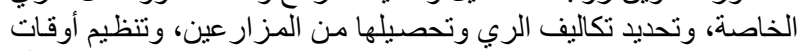

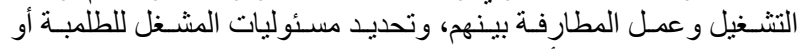

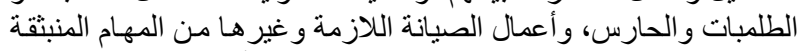

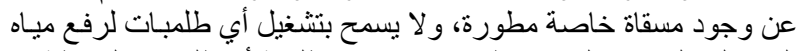

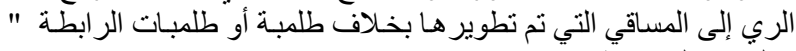

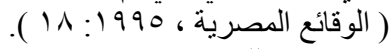

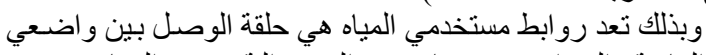

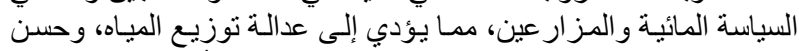

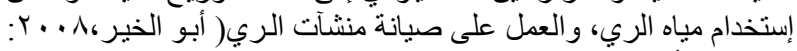

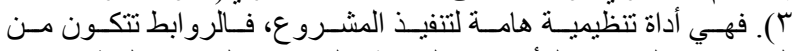

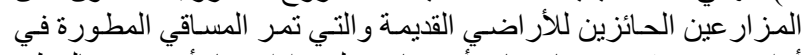

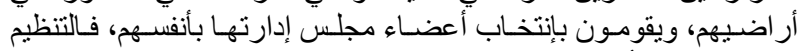

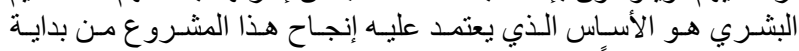

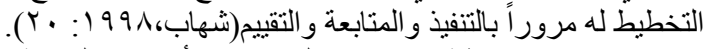

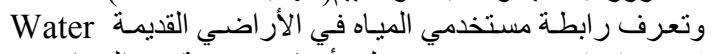

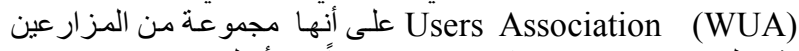

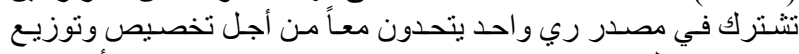

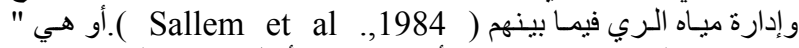

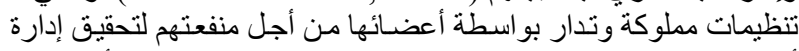

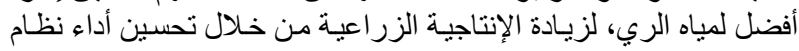

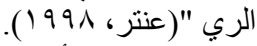

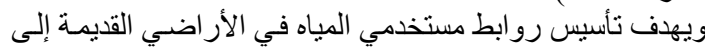

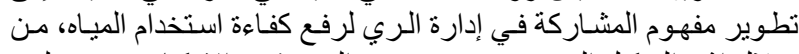

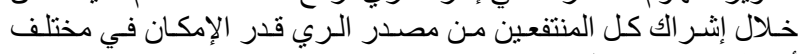

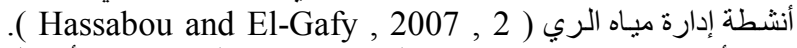

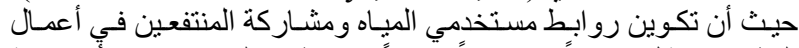

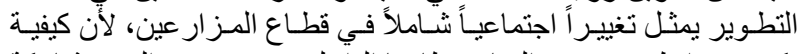

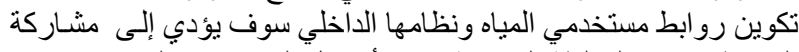

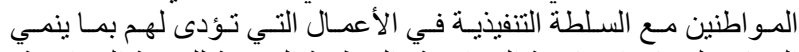

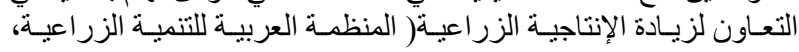

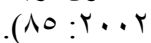

ونظر اً لأن محافظة كفر الثيخ من أولى المحافظات التي نفذ فيها التهاء

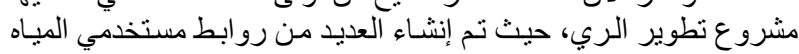

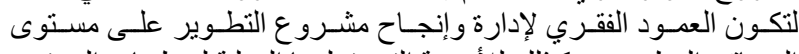

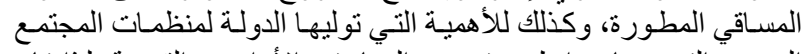

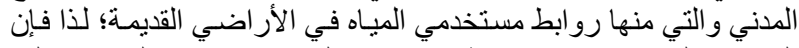

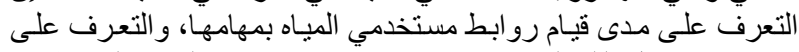

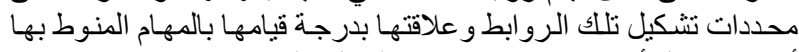

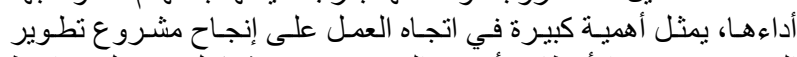

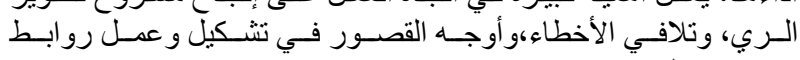
مستخدمي المياه. وتنطرح هذه الدر اسة تساؤلاً مؤداه: ما مدى قيام رو ابط مستخدمي

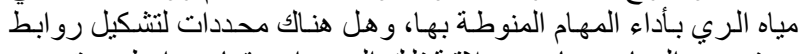

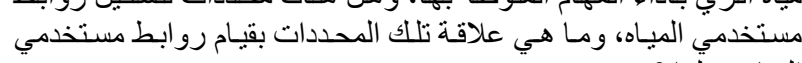

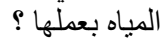
الأهداف البحثية : البعاه ا ـالتعرف على مستوى قيام رو ابط مستخدمي المياه بمهامها.

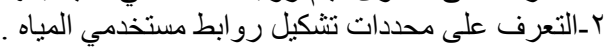

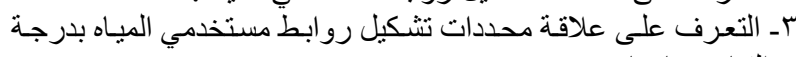
القيام بمهامها. 


\begin{tabular}{|c|c|c|}
\hline$\%$ & 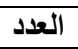 & نتوى قيام روابط مستخدمي المياه بمهامها \\
\hline $0, Y, Y$ & IT & خفض(IV_ ( I ) درجة \\
\hline ro, r r & $0 \wedge$ & وسط (1) ( \\
\hline $79,0 \mathrm{~V}$ & 17. & 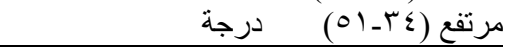 \\
\hline
\end{tabular}

\begin{tabular}{|c|c|c|c|c|c|c|c|c|c|}
\hline \multirow{2}{*}{ الترتيب } & \multirow{2}{*}{ المرجح } & \multicolumn{2}{|c|}{ نادراً } & \multicolumn{2}{|c|}{ أحيانا } & \multicolumn{2}{|c|}{ دائماً } & \multirow{2}{*}{\multicolumn{2}{|c|}{ البنود }} \\
\hline & & $\%$ & 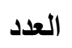 & $\%$ & 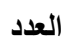 & $\%$ & 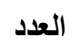 & & \\
\hline 1 & $r, r \cdot$ & $1 \leqslant, \vee \wedge$ & $r \varepsilon$ & $\varepsilon \cdot \wedge \vee$ & $9 \leq$ & $\varepsilon \leqslant, r_{0}$ & $1 \cdot r$ & التعاون مع مهندس التوجيه المائي في الإعداد للمشروع & \\
\hline$\varepsilon$ & r, r & $1 T, \varepsilon$ & $r \cdot$ & $\leqslant 9, \circ \mathrm{V}$ & $11 \leqslant$ & $r v, r q$ & $\wedge \mathrm{r}$ & التعاون مع المهندس التنفيذي في تِنفيَّ المشروع & ( \\
\hline IT & r, 10 & $1 \leqslant, r_{0}$ & 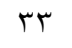 & $07,0 Y$ & Ir. & $r q, 1 r$ & TV & استلام المسّقى بعد تطوير هاً & $Y$ \\
\hline 1. & r, IV & 14,91 & rt & $00, Y Y$ & IrV & $r \cdot, \wedge v$ & (1) & تحديد مسئولية الآلة و المسقى & \\
\hline 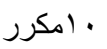 & r,iv & $r \cdot, \varepsilon r$ & $\leqslant \vee$ & $\varepsilon r, I V$ & $9 \vee$ & $r v, r q$ & $\wedge 7$ & وضع الخطة السنوية للصيانة و التشغيل & $c$ \\
\hline 10 & $r, \cdot T$ & $1 \cdot, \leqslant r$ & $r \leq$ & $\pi$ & $1 \leq 0$ & $r, r$. & $\leq 9$ & تنظيم الاجتماعات الدورية لتنظيم سير العمل & \\
\hline ع مكرر & r,tr & $1 \cdot, \varepsilon r$ & $r \leq$ & 07,9 & 149 & N & VV & التنسيق مع الرو ابط الأخرى لتنسيق المناوبات & $y$ \\
\hline 9 & $r, 1 \wedge$ & $r \cdot, \varepsilon r$ & $\leqslant \vee$ & $\varepsilon \cdot, \wedge \vee$ & $9 \leq$ & rی, v. & $\wedge 9$ & وضع أسس المحاسبة المالية و إدارتها & \\
\hline r & r, rq & $\Lambda, Y_{\top}$ & 19 & $0 \leqslant, \leqslant \wedge$ & $1 T^{1}$ & $r v, \ldots$ & 10 & تحديد بنود ميز انية الر ابطة & \\
\hline r & $r, r \varepsilon$ & IV,Ar & 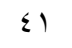 & $\varepsilon$. & 94 & $\varepsilon r, I V$ & $9 \vee$ & ا تحديد أسلوب تحصيل الموارد المالية اللازمة للرابطة & \\
\hline$\wedge$ & $r, r_{1}$ & r), & $\leqslant 9$ & ry,or & $\wedge \varepsilon$ & $\varepsilon r, I V$ & $9 \vee$ & عرض الأمور المالية على أعضاء الر ابطة & 1 \\
\hline $\mathrm{v}$ & r,tr & $r \cdot, 9$. & $\varepsilon \wedge$ & $r, \cdot 9$ & $\lambda r$ & $\varepsilon \mu^{\prime}, \varepsilon$ & 99 & ا حل المشاكل التي تنشأ بين الأعضاء & $T$ \\
\hline 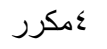 & r, & 17,99 & $r v$ & $\leqslant 0, r\}$ & $1 \cdot \varepsilon$ & $r \wedge, v$. & $\wedge 9$ & ' وضع القرار الحَّاسم لفض أب نزاع ينشأ بين الزراع & Ir \\
\hline $1 \varepsilon$ & $r, \cdot \wedge$ & $r), V \varepsilon$ & 0 . & $\leqslant \Lambda, \nu$. & $11 \mathrm{r}$ & $r q, 0 V$ & 71 & نشر الو عي اللازم للمحافظة على البيئة & \\
\hline iv & $r, \cdots$ & IV,Ar & 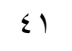 & 74,91 & $1 \leq V$ & $1 \wedge, Y_{7}$ & $\varepsilon r$ & نشر الوعي اللازم للمحافظة على مياه الري & 10 \\
\hline م امكرر & $r, \cdot r$ & $11, Y_{7}$ & $\leqslant r$ & OV, & שr" & $r r, q)$ & 00 & & 11 \\
\hline 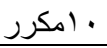 & r,IV & 17,9 & rv & $01, \mu$. & 111 & rr, TI & vo & نقل أي خبر ات جديدة بخصوص المشروع & \\
\hline
\end{tabular}

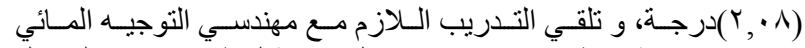

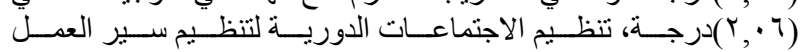

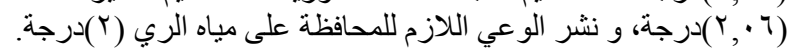

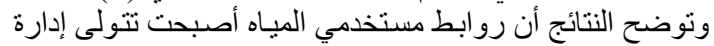

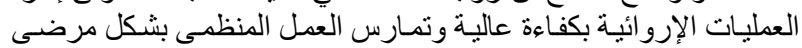

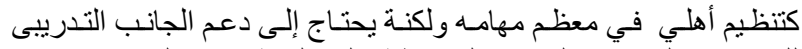

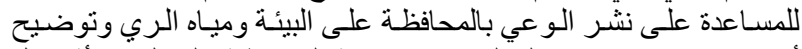

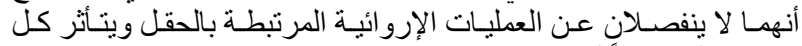

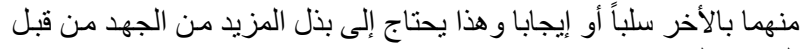

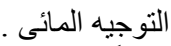
ثانيـاً: النتـائج التـي تتعلى بالأهميـة النسـبية لمحددات تثـكيل روابط

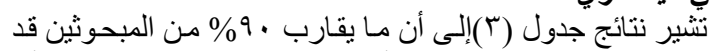

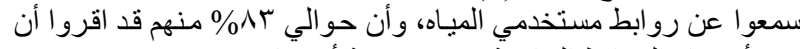

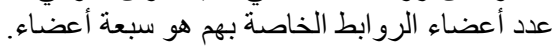

وبثـى أكثر تقصيلاً تبـين نتائج جدول (r) الأهميـة النسبية

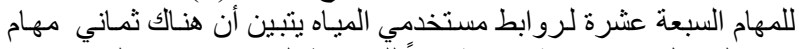

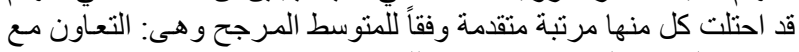

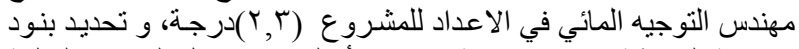

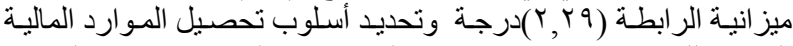

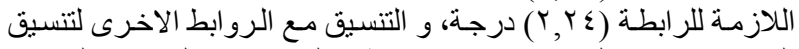

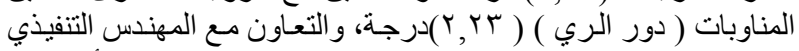

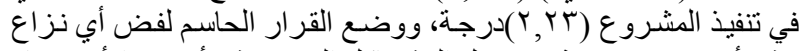

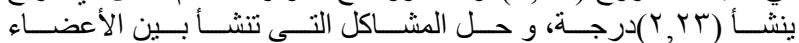

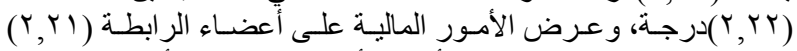

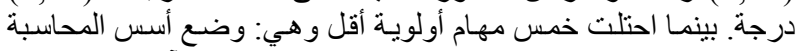

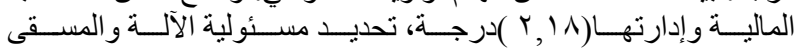
(Y, IV)

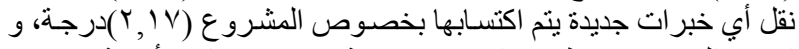

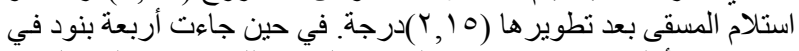

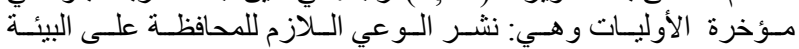

جدول (r) التوزيع والنسبة المئوية للمبحوثين وفقا للسماع عن روابط مستخدمي المياه، وعدد أعضاء الرابطة

\begin{tabular}{|c|c|c|c|}
\hline$\%$ & عدد & الاستجابات & السماع عن الرابطة، وعدد أعضاء الرابطة \\
\hline $19,0 \mathrm{~V}$ & $r \cdot T$ & نعم & \\
\hline $1 \cdot, \varepsilon r$ & $r \varepsilon$ & $y$ & 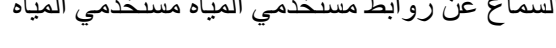 \\
\hline 17,97 & rq & م أعضاء & \\
\hline$\Lambda r, \varepsilon$ & 191 & V أعضاء V & عدد اعضاء الر ابطه \\
\hline
\end{tabular}

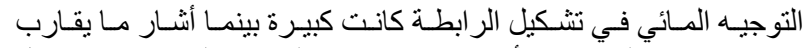

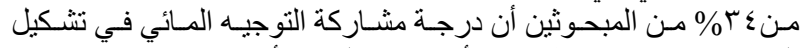

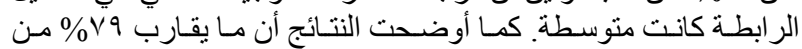

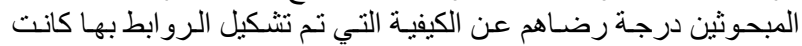

متوسطة.

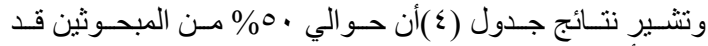

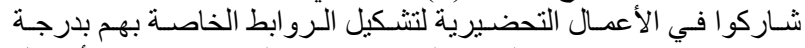

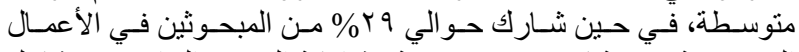

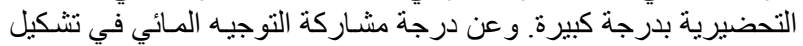

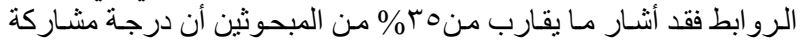


Hegazy, H. M. E.

جدول(ع): التوزيع والنسبة المئوية للمبحوثين وفق محددات تثكيل الرابطة

\begin{tabular}{|c|c|c|c|c|c|}
\hline \multicolumn{2}{|c|}{ ע } & \multicolumn{2}{|c|}{ بلرجة متوسطة } & \multicolumn{2}{|c|}{ بلرجة كبيرة } \\
\hline$\%$ & عدد & $\%$ & عدد & $\%$ & عدد \\
\hline$r Y, T I$ & or & $\sum 9,14$ & $11 \pi$ & $r \Lambda, Y \uparrow$ & 70 \\
\hline rY,IV & $V \varepsilon$ & A & $V V$ & $r \leqslant, r_{0}$ & $v q$ \\
\hline 10,70 & דוץ & $\vee \wedge, \vee$. & $\mid 11$ & 0,70 & Tr \\
\hline I & r. & ור. & $1 \leqslant V$ & rr. & or \\
\hline$r \cdot, \cdots$ & $\leq 7$ & $\leq 7,97$ & $1 \cdot 1$ & s & VT \\
\hline rq, OV & 71 & $\varepsilon \varepsilon, \vee \wedge$ & $1 . r$ & Y0,70 & 09 \\
\hline$r \cdot, \cdots$ & $\leqslant 7$ & $v \leqslant, r_{0}$ & $|v|$ & 0,70 & Tr \\
\hline
\end{tabular}

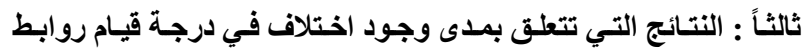

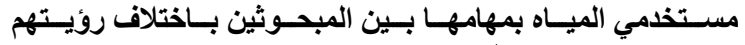

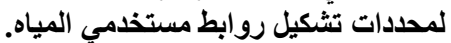

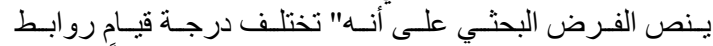

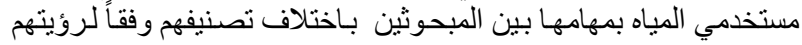

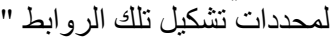

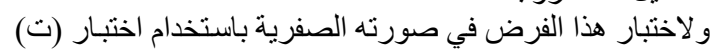

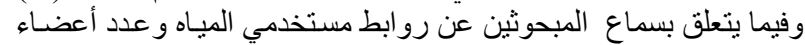

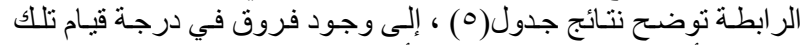

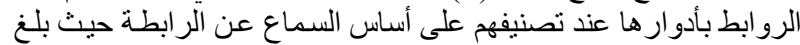

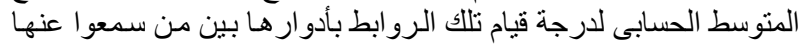

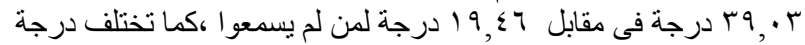

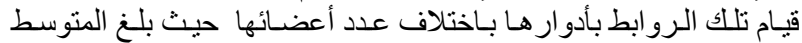

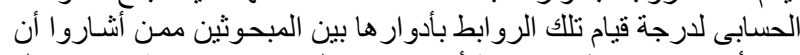

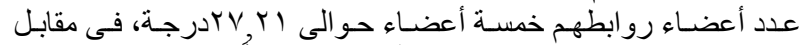

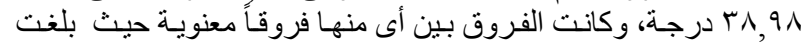

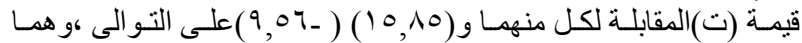

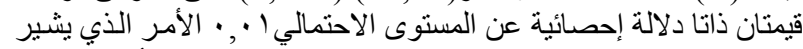

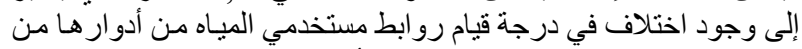

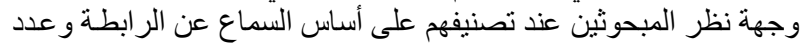

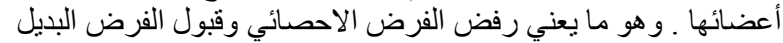

محددات تثثكيل الرابطة

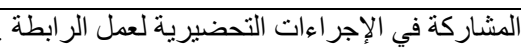
مشاركة التوجيه المائي في تشكيل الرابطة الرئة الرضا عن الكيفية التى شكلت بهان بها الر ابطة.

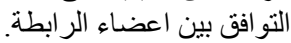

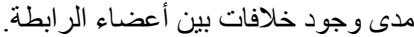

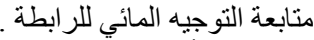
الرضا عن أسلوب الَّعمل بالربطة البطة

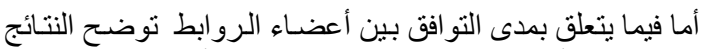

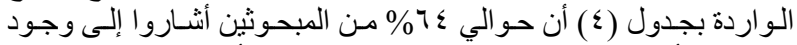

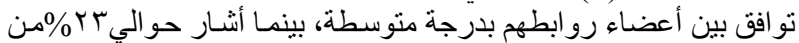

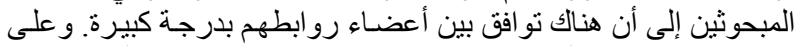

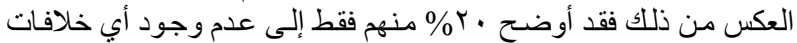

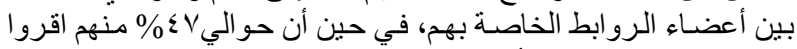

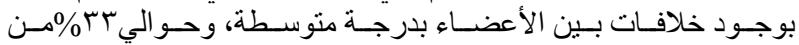

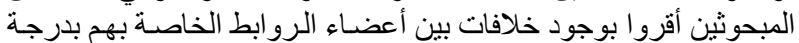

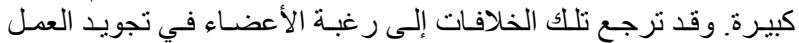

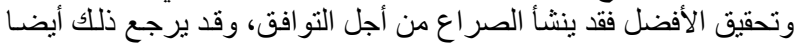

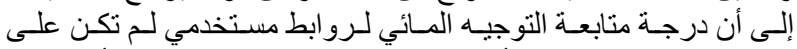

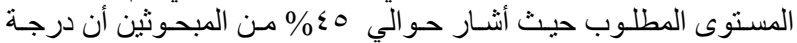

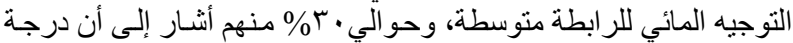

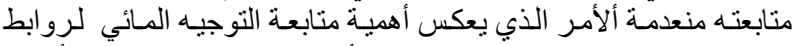

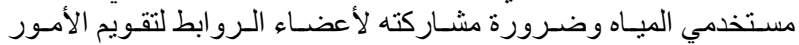

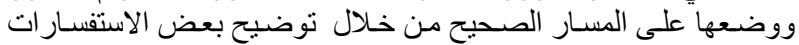

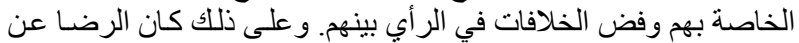

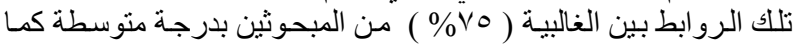
بينت النتائج بنفس الجدول.

جدول(0): نتائج اختبار (ت) لاختبار معنوية الفرق في درجة قيام روابط مستخدمي المياه بمهامها بين المبحوثين عند تصنيفهم على أسـاس درجة

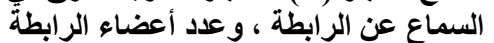

\begin{tabular}{|c|c|c|c|c|c|}
\hline قيمة (ت) & الانحراف المعياري & المتوسط الحسابي & ن & الاستجابات & محددات تثكيل الرابطة \\
\hline \multirow{2}{*}{$* * 10,10$} & 0,90 & $r q, \cdot r$ & $r \cdot T$ & نعم & \multirow{2}{*}{ السماع عن رو ابط مستخدمي المياه مستخذمي المياه } \\
\hline & $r, \cdot 1$ & $19, \leqslant 7$ & $r \leqslant$ & $\gamma$ & \\
\hline \multirow{2}{*}{$* * 9.07$} & 11,79 & $r v, Y)$ & rq & خمسة أعضاء & \multirow{2}{*}{ عدد أعضاء الرابطة } \\
\hline & $0.7 \pi$ & $r \wedge, q \Lambda$ & 191 & سبعة أعضاء & \\
\hline
\end{tabular}

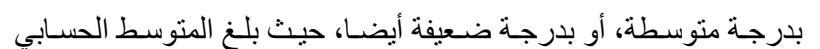

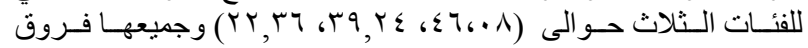

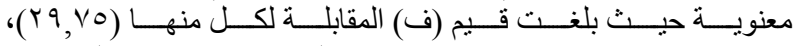

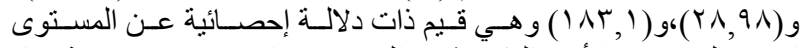

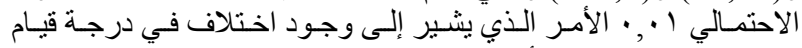

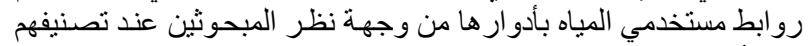

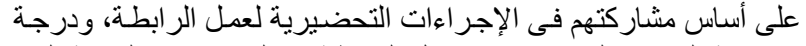

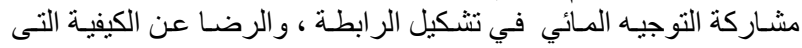

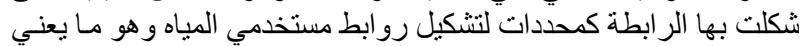
رفض الفرض الاحصائي وقبول الفرات لفيل روالبـ البديل.

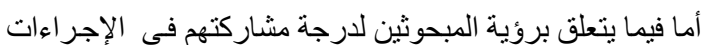

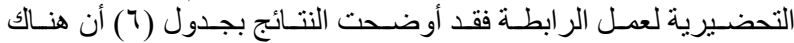

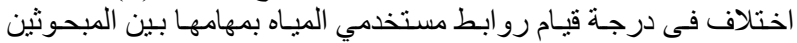

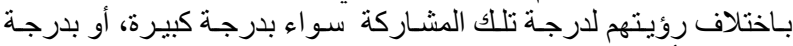

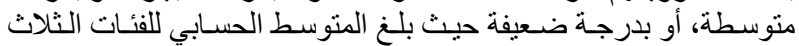
حو الى (r,T

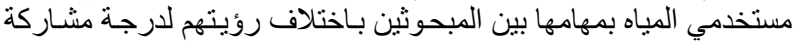

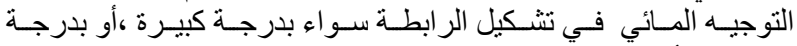

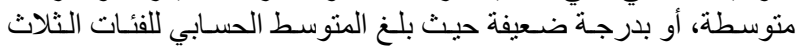

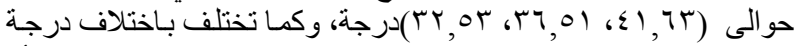

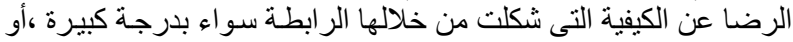

جدول(7): نتائج اختبار (ف) لاختبار معنوية الفروق في رؤية المبحوثين لمتوسطات درجة قيام روابط مستخدمي المياه بمهامها عند تصنيفهر على

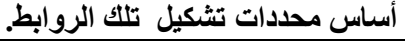

\begin{tabular}{|c|c|c|c|c|}
\hline \multirow{2}{*}{ قيمة (ف) } & \multicolumn{3}{|c|}{ متوسطات درجة قيام روابط مستخدمى المياه بمهامها. } & \multirow{2}{*}{ محددات تثكيل الرابطة } \\
\hline & بلرجة ضعفة & بل برجة متوسطة & بل لدرجة كبيرة & \\
\hline$* * r_{q}, \vee_{0}$ & $r$ r,O & $r 0, v 9$ & $\varepsilon r, 7 \mu$ & المشاركة في الإجر اءات التحضيرية لعمل الر ابطة . \\
\hline$* * \curlyvee \wedge, q \wedge$ & rr,or & 47,01 & «1, ז & مشاركة التوجيه المائي في تشكيل الر ابطة . \\
\hline **।人ז, ,. & $r, \xi$. & rq, r & $\leqslant 7,1$ & الرضا عن الكيفية التى" شكتّت بها الر ابطة. \\
\hline$* * r .0,11$ & $19, \mathrm{VV}$ & 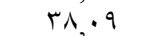 & $\varepsilon r, 7 \Lambda$ & التو افق بين أعضاء الربطة. \\
\hline$* * 1,7,1$. & ro, 10 & $r \Lambda, \leqslant 0$ & $\varepsilon 1,7 \varepsilon$ & مدى وجو د خلافات بين أعضاء الر ابطة. \\
\hline$* * r \varepsilon, 9 r$ & $r \varepsilon, \varepsilon q$ & r r r & $\varepsilon r, q r$ & متابعة التوجيه المائي للر ابطة. \\
\hline$* * \wedge q, \vee 1$ & $r 7, \leqslant \Lambda$ & $r q, i r$ & $\varepsilon 7,1$ & الرضا عن أسلوب الَعمل بالر ابطة \\
\hline
\end{tabular}




\section{J. Agric. Econom. and Social Sci., Mansoura Univ., Vol.7 (3), March ,2016}

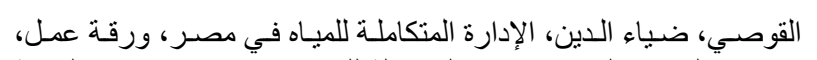

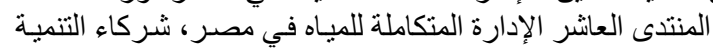

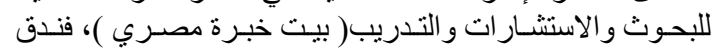

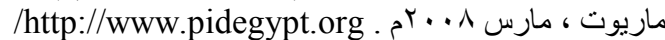

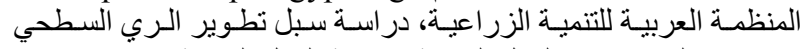

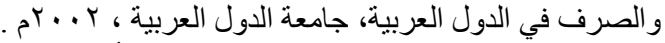

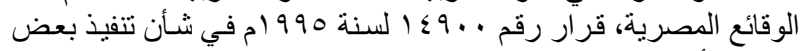

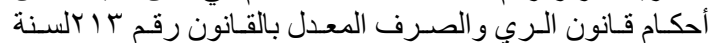

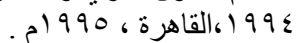

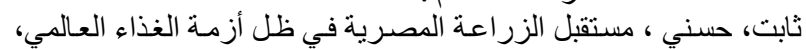

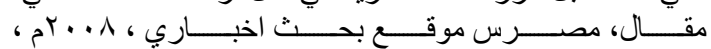
/http://www.masress.com

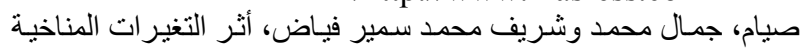

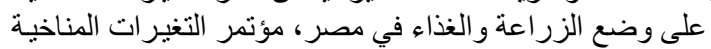

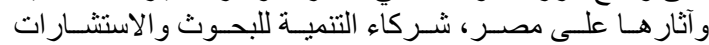

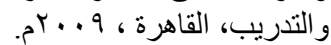

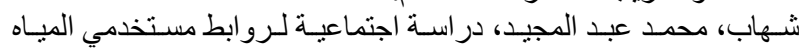

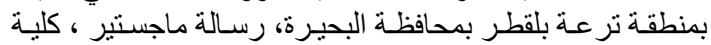

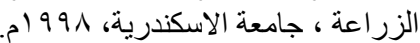

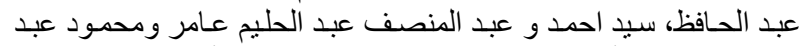

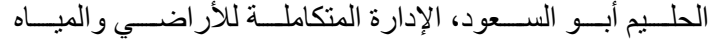

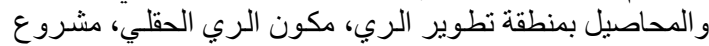

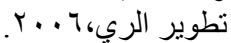

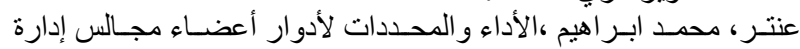

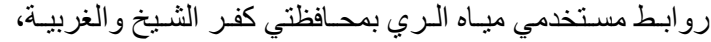

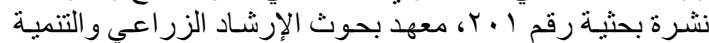

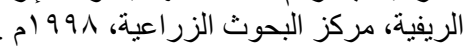

FAO ,2007 , Egypt's Experience in Irrigation and Drainage Research Uptake, Final Report, Rome , 2007 .

Gad, Abd - Alla and Raffat Ramadan Ali, Water rationalization in Egypt from the perspective of the virtual water concept, Options Méditerranéennes: Série A. Séminaires Méditerranéens; n. 88, p.301- 310, 2009.

Hassabou , Abdel Hakim M., Inas K . El-Gafy , Assessment Indicators For Water Users' Association in Egypt, Official Publication, The European Water association, 2007.

Sallem M., Naguib M., Saber A., El-Masry A., El-Attar A., Ezz El-dine E., Abdel Al-Omar F., Hassanein F., Dweeb H., and Youssef S., Experience With Water User's Association , EWUP Technical Report No. 65, Water Use and Management Project , Ministry of Water Resources and Irrigation, Egypt, 1984 .

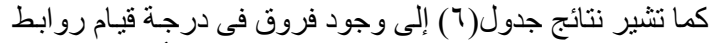

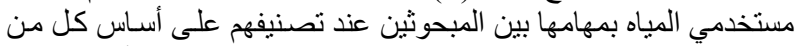

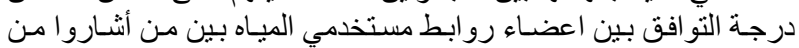

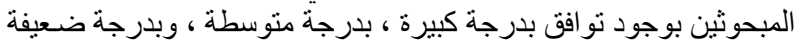

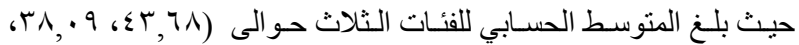

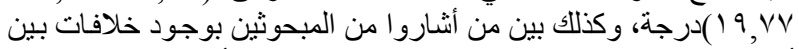

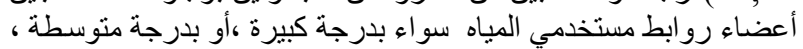

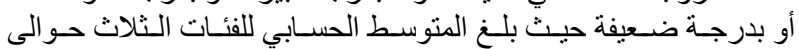

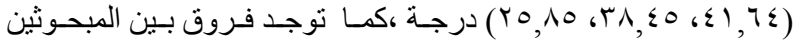

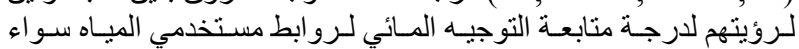

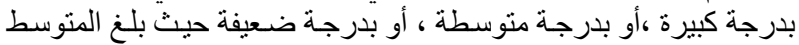

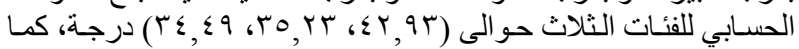

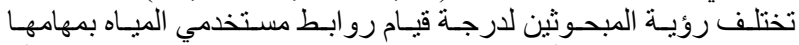

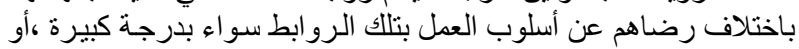

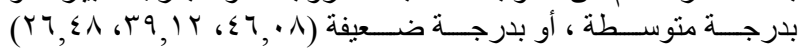

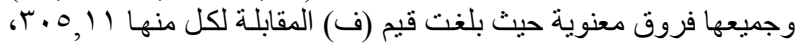

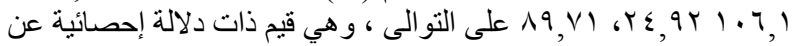

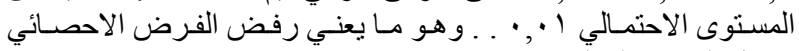

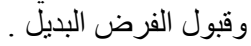
الأهمية التطبيقية:

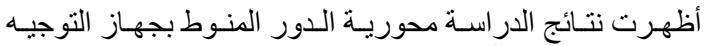

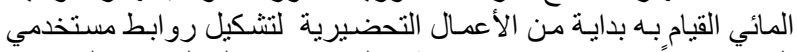

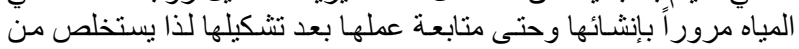

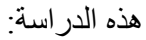

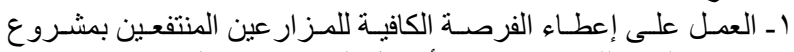

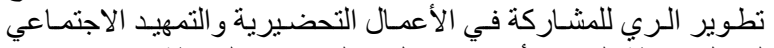

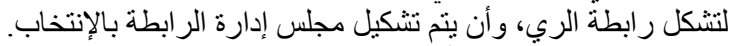

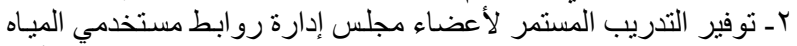

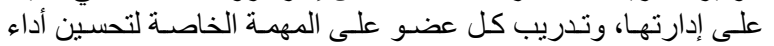

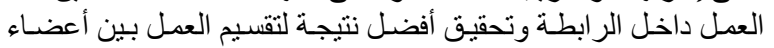

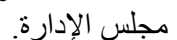

rـ ضرورة توفير التدريب المناسب لأعضاء رواء روابط مستخدمي المياه في

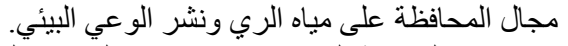

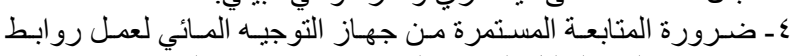

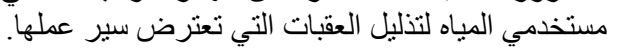

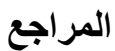

أبو الخير، منير يوسف سيد أحمد، ترشيد إستخدام مياه الري، دراسـة حالة

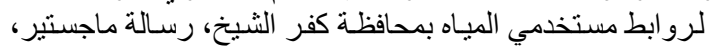

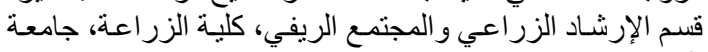

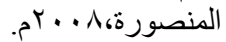

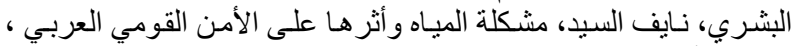

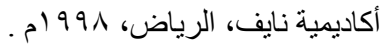


Hegazy, H. M. E.

DETERMINANTS OF WATER USERS ASSOCIATION FORMATION AND THEIR RELATIONSHIP WITH CARRYING OUT ITS TASKS, AFIELD STUDY ON ELMANAIFA CANAL FARMERS AT KAFER EL-SHIEKH GOVERNORATE

Hegazy, H. M. E.

Agric. Extension \& Rural Development Research Inst . ARC

\begin{abstract}
This research aimed to identify the formation determinants of WUAs and carrying out its tasks. To achieve this objective it should be throw: identifying the level of the water users associations tasks, identifying the formation of WUAs determinants, and to identify the relationship of the determinants of user associations water formation and carrying out its tasks from the perspective of the beneficiaries of irrigation improvement project ( IIP ); to achieve these goals, research was conducted on El-manaifa canal at Kafer El-Sheikh governorate which include with irrigation improvement project ( IIP ); by a sample totaling 230 respondents.

Data were collected by personal interview throw questionnaire in order to achieve the research goals after tested initially and validated as a tool to collect the data ; many statistical methods were used such as frequencies, percentages, and the arithmetic mean, standard deviation, and both the( $\mathrm{T}$ test and $\mathrm{F}$ test were to analyze the data.

The most fiddling of this research were as follows :

- The level of the water users associations duties was high as indicated by the 160 respondents representing about $70 \%$ of them.

- There are eight tasks each of which has occupied an advanced position in accordance with the weighted degree , namely: cooperation with the Water Directive engineer in the preparation of the project, determine the Association's budget items, determine the collection of the necessary financial association resource method, and coordination with other links to coordinate rotations, cooperate with the Executive Engineer in the implementation of the project degrees ,place the crucial decision to settle any dispute arising out of and the solution problems that arise between members of and the presentation of financial matters to members of the League.

- that there are four tasks each of which had occupied a back seat: the deployment of the necessary awareness to preserve the environment, receive the necessary training with water directive engineers, organizing regular meetings to organize the workflow, and deploy the necessary awareness to conserve irrigation water.

- There are significant differences in the degree of water users associations tasks among respondents when classified according to the formation determinants of WUAs.
\end{abstract}

